\title{
Application of Quasisubordination to Certain Classes of Meromorphic Functions
}

\author{
Syed Ghoos Ali Shah $\mathbb{D}^{1}$, Saqib Hussain $\mathbb{D}^{1},{ }^{1}$ Akhter Rasheed, ${ }^{1}$ Zahid Shareef, ${ }^{2}$ \\ and Maslina Darus ${ }^{3}$
}

${ }^{1}$ Department of Mathematics, COMSATS University Islamabad, Abbottabad Campus, 22060, Pakistan

${ }^{2}$ Mathematics and Natural Science, Higher Colleges of Technology, Fujairah Men's, Fujairah 4114, UAE

${ }^{3}$ Department of Mathematical Sciences, Universiti Kebangsaan Malaysia, Bangi, 43600 Selangor, Malaysia

Correspondence should be addressed to Saqib Hussain; saqib_math@yahoo.com

Received 2 July 2020; Revised 12 September 2020; Accepted 25 September 2020; Published 14 October 2020

Academic Editor: Fanglei Wang

Copyright (C) 2020 Syed Ghoos Ali Shah et al. This is an open access article distributed under the Creative Commons Attribution License, which permits unrestricted use, distribution, and reproduction in any medium, provided the original work is properly cited.

Inequalities play a fundamental role in many branches of mathematics and particularly in real analysis. By using inequalities, we can find extrema, point of inflection, and monotonic behavior of real functions. Subordination and quasisubordination are important tools used in complex analysis as an alternate of inequalities. In this article, we introduce and systematically study certain new classes of meromorphic functions using quasisubordination and Bessel function. We explore various inequalities related with the famous Fekete-Szego inequality. We also point out a number of important corollaries.

\section{Introduction}

A complex valued function is said to be meromorphic if it has poles as its only singularities. Let $\sum_{1}$ denotes the class of all of meromorphic functions which has a simple pole at $\omega=0$ and has Laurent series expansion of the form:

$$
\lambda(\omega)=\frac{1}{\omega}+\sum_{t=0}^{\infty} a_{t} \omega^{t}
$$

which are analytic in the punctured open unit disc $U^{*}=\{\omega$ $: \omega \in \mathrm{C}$ and $0<|\omega|<1\}=U-\{0\}$, as open unit disc $U=U^{*}$ $\cup\{0\}$.

Here, we are listing some important subclasses of meromorphic functions which will be used in our subequal work. In 1936, Robertson [1] introduced the classes of meromorphic starlike and meromorphic convex functions of order $\alpha$. By $\sum^{M S}(\alpha)$, we mean the subclass of $\sum_{1}$ consisting of all meromorphic starlike functions of order $\alpha$. Analytically,

$$
\lambda(\omega) \in \sum^{M S}(\alpha) \Leftrightarrow \operatorname{Re}\left(\frac{\omega \lambda^{\prime}(\omega)}{\lambda(\omega)}\right)<-\alpha,\left(0 \leq \alpha<1 ; \omega \in U^{*}\right) .
$$

A closely related class of meromorphic convex functions of order $\alpha$ is denoted by $\sum^{M C}(\alpha)$ and defined as

$$
\lambda(\omega) \in \sum^{M C}(\alpha) \Leftrightarrow-\omega \lambda^{\prime}(\omega) \in \sum^{M S}(\alpha) .
$$

In 1952, W. Kaplan [2] introduced and studied an important class of analytic functions known as close-to-convex functions in the open unit disc $U$. A function $\lambda$ belongs to $\sum_{1}$, is in class $\sum^{M C}(\alpha, \beta)$, of meromorphic close-to-convex functions of order $\alpha$ and type $\beta$ if there exist $\delta(\omega) \in \sum^{M S}(\beta)$, and

$$
\operatorname{Re}\left(\frac{\omega \lambda^{\prime}(\omega)}{\delta(\omega)}\right)<-\alpha
$$


Let $\delta(\omega) \in \sum_{1}$ and having series representation of the form

$$
\delta(\omega)=\frac{1}{\omega}+\sum_{t=0}^{\infty} b_{t} \omega^{t}
$$

Then, the convolution of $\lambda$ and $\delta$ as denoted by $\lambda * \delta$ is defined as

$$
(\lambda * \delta)(\omega)=\frac{1}{\omega}+\sum_{t=0}^{\infty} a_{t} b_{t} \omega^{t}=(\delta * \lambda)(\omega)
$$

where $\lambda$ is given by (1).

A function $\lambda$ is subordinate to $\delta$ in $U^{*}$ and written as $\lambda$ $(\omega) \prec \delta(\omega)$, if there exists a Schwarz function $k(\omega)$, which is holomorphic in $U$ with $k(0)=0$, such that $\lambda(\omega)=\delta(k(\omega))$. Let $\phi(\omega)$ be an analytic function with positive real part on $U$ satisfies $\phi(0)=1$ and $\phi^{\prime}(0)>0$ which maps $U$ which is star shape with respect to $\omega=1$, also symmetric with respect to the real axis. We denote $\sum(\phi)$ be the class of function $\lambda \in \sum_{1}$ for which $-\omega \lambda^{\prime}(\omega) / \lambda(\omega) \prec \phi(\omega),\left(\omega \in U^{*}\right)$. The class $\sum(\phi)$ was introduced and studied by Silverman et al. [3] (see also [4]). The class $\sum(\alpha)$ is a special case of the class $\sum(\phi)$ when $\phi(\omega)=1+(1-2 \alpha) \omega / 1-\omega(0 \leq \alpha<1)$.

Robertson [5] gave the idea of quasisubordination. For any two functions $\lambda(\omega)$ and $\delta(\omega)$, holomorphic in $U$, the function $\lambda(\omega)$ is said to be quasi-subordinate to the function $\delta(\omega)$ written as $\lambda(\omega) \prec_{q} \delta(\omega)$, if there exists two holomorphic functions $\lambda(\omega)$ and $\varphi(\omega)$, with $|\varphi(\omega)| \leq 1, \lambda(\omega) / \varphi(\omega)$ is holomorphic in $U^{*}$ and such that $\lambda(\omega)=\varphi(\omega) \delta(k(\omega))$. In particular, if $\varphi(\omega)=1$, then quasisubordination reduces to subordination. Furthermore, if $k(\omega)=1$, the quasisubordination becomes the majorization, (see [6]), which implies

$\lambda(\omega) \prec_{q} \delta(\omega) \Rightarrow \lambda(\omega)=\varphi(\omega) \delta(\omega) \Rightarrow \lambda(\omega)<<\delta(\omega)\left(\omega \in U^{*}\right)$

For recent work on meromorphic functions, we refer [7-18].

Motivated from the above cited work, we introduce the following subclasses of meromorphic functions. Throughout in this paper, we shall assume $0 \leq \gamma<1, \gamma \neq 1 / 2,0 \leq \eta<1, \omega$ $\in U^{*}, \lambda, \delta \in \sum_{1}$, and $\phi(\omega)$ be an analytic function with positive real part on $U$ that satisfies $\phi(0)=1$ and $\phi^{\prime}(0)>0$ which maps $U$ which is star shape with respect to $\omega=1$, and also symmetric with respect to the real axis unless otherwise mentioned.

Definition 1. Let $\sum_{q}^{M C}(\phi, \gamma)$ be the class of functions $\lambda(\omega) \epsilon$ $\sum_{1}$ and satisfy

$-\left[(1-\gamma)\left(\frac{\omega \lambda^{\prime}(\omega)}{\lambda(\omega)}\right)+\gamma\left(\left(1+\frac{\omega \lambda^{\prime \prime}(\omega)}{\lambda^{\prime}(\omega)}\right)\right]-1 \prec_{q} \phi(\omega)-1, \quad\left(\omega \in U^{*}\right)\right.$.

The abovementioned class $\sum_{q}^{M C}(\phi, \gamma)$ is the meromorphic analogue of the class $S_{q}(\phi)$ introduced and studied by
Mohd and Darus [19]. For $\gamma=0$, the class $\sum_{q}^{M S}(\phi)$ was studied by Zayed et al. [20].

Definition 2. Let $\sum_{q}^{M K}(\phi, \eta)$ be the subclass of $\sum_{1}$ consisting of all functions $\lambda(\omega)$ for which their exist $\delta(\omega) \in \sum^{M S}(\alpha)$ and satisfy

$$
\frac{-\omega \lambda^{\prime}(\omega)}{(1-\eta) \delta(\omega)+\eta \omega \delta^{\prime}(\omega)}-1 \prec_{q} \phi(\omega)-1, \quad\left(\omega \in U^{*}\right) .
$$

For $\eta=0$ and $\delta(\omega)=\lambda(\omega)$, the class $\sum_{q}^{M S}(\phi)$ was studied by Zayed et al. [20].

In this paper, we obtain the Fekete-Szego inequality for meromorphic functions belonging to above defined classes. Let $k \in \Omega$ denote the class of functions of the form $k(\omega)=$ $k_{1} \omega+k_{2} \omega^{2}+k_{3} \omega^{3}+\cdots$, satisfying $|k(\omega)|<1$, for $\omega \in U^{*}$. For more details, see [21-23]. To prove our main results, we need the following lemma.

Lemma 3 [24]. If $k \in \Omega$, then for any complex number $u, \mid k_{2}$ $-u k_{1}^{2} \mid \leq \max \{1 ; u\}$, the result is sharp for the functions given by $k(\omega)=\omega \operatorname{or} k(\omega)=\omega^{2}$.

\section{Main Results}

In this section, we explore certain Fekete-Szego-related inequalities for the class $\sum_{q}^{M C}(\phi, \gamma)$ and $\sum_{q}^{M K}(\phi, \eta)$.

Theorem 4. Let $\phi(\omega)=1+B_{1} \omega+B_{2} \omega^{2}+\cdots, B_{1}>0$, and $\varphi(\omega)=c_{0}+c_{1} \omega+c_{2} \omega^{2}+\cdots$ if $\lambda(\omega)$ given by (1) be in the class $\sum_{q}^{M C}(\phi, \gamma)$, and $\mu$ is a complex number, then

$\left|a_{1}-\mu a_{0}^{2}\right| \leq \frac{B_{1}}{2(1-2 \gamma)}\left[1+\max \left\{1,\left|\frac{B_{2}}{B_{1}}\right|+B_{1}\left|\frac{1}{1-\gamma}-2 \mu \frac{(1-2 \gamma)}{(1-\gamma)^{2}}\right|\right\}\right]$.

The inequalities are sharp for $k(\omega)=\omega$ or $k(\omega)=\omega^{2}$.

Proof. Let $\lambda(\omega) \in \sum_{q}^{M C}(\phi, \gamma)$, then there exist analytic functions $\varphi(\omega)$ and $k(\omega)$, with $|\varphi(\omega)|<1, k(0)=0$, and $k(\omega)<1$ such that

$$
\begin{gathered}
-\left[(1-\gamma)\left(\frac{\omega \lambda^{\prime}(\omega)}{\lambda(\omega)}\right)+\gamma\left(1+\frac{\omega \lambda^{\prime \prime}(\omega)}{\lambda^{\prime}(\omega)}\right)\right]-1 \\
=\varphi(\omega)[\phi(k(\omega))-1], \quad\left(\omega \in U^{*}\right) .
\end{gathered}
$$

Taking first and second derivative of (1), and use in the left hand side of above equation, we obtain 


$$
\begin{aligned}
& -\left[(1-\gamma)\left(\frac{\omega \lambda^{\prime}(\omega)}{\lambda(\omega)}\right)+\gamma\left(1+\frac{\omega \lambda^{\prime \prime}(\omega)}{\lambda^{\prime}(\omega)}\right)\right] \\
& =(1-\gamma)\left[1-a_{0} \omega+\left(a_{0}^{2}-2 a_{1}\right) \omega^{2}\right]+\gamma\left(1+2 a_{1} \omega^{2}\right)+\cdots
\end{aligned}
$$

then implies

$$
\begin{gathered}
-\left[(1-\gamma)\left(\frac{\omega \lambda^{\prime}(\omega)}{\lambda(\omega)}\right)+\gamma\left(1+\frac{\omega \lambda^{\prime \prime}(\omega)}{\lambda^{\prime}(\omega)}\right)\right]-1 \\
=-a_{0}(\omega)(1-\gamma)+\left[\left(a_{0}^{2}-2 a_{1}+\gamma\left(-a_{0}^{2}+4 a_{1}\right)\right] \omega^{2}+\cdots\right. \\
\phi(k(\omega))=\phi\left(k_{1} \omega+k_{2} \omega^{2}+k_{3} \omega^{3}+\cdots\right) \\
=1+k_{1} B_{1} \omega+\left(k_{1}^{2} B_{2}+k_{2} B_{1}\right) \omega^{2} \\
\quad+\left(k_{3} B_{1}+2 k_{1} k_{2} B_{2}+k_{1}^{3} B_{3}\right) \omega^{3}+\cdots
\end{gathered}
$$

then

$$
\begin{aligned}
\varphi(\omega)[\phi(k(\omega))-1]= & {\left[c_{0}+c_{1} \omega+c_{2} \omega^{2}+\cdots\right] } \\
& \cdot\left[1+k_{1} B_{1} \omega+\left(k_{1}^{2} B_{2}+k_{2} B_{1}\right) \omega^{2}\right. \\
& \left.+\left(k_{3} B_{1}+2 k_{1} k_{2} B_{2}+k_{1}^{3} B_{3}\right) \omega^{3}+\cdots\right],
\end{aligned}
$$

which implies

$\varphi(\omega)[\phi(k(\omega))-1]=c_{0} k_{1} B_{1} \omega+\left(c_{0} k_{1}^{2} B_{2}+c_{0} k_{2} B_{1}+c_{1} k_{1} B_{1}\right) \omega^{2}+\cdots$.

Comparing (13) and (16), we get

$$
\begin{gathered}
a_{0}=-\frac{c_{0} k_{1} B_{1}}{1-\gamma} \\
a_{1}=-\frac{B_{1} c_{0}}{2(1-2 \gamma)}\left[k_{2}+k_{1} \frac{c_{1}}{c_{0}}+k_{1}^{2}\left(\frac{B_{2}}{B_{1}}-\frac{B_{1} c_{0}}{(1-\gamma)}\right)\right] .
\end{gathered}
$$

Thus,

$a_{1}-\mu a_{0}^{2}=-\frac{B_{1} c_{0}}{2(1-2 \gamma)}\left[k_{2}+k_{1} \frac{c_{1}}{c_{0}}+k_{1}^{2}\left(\frac{B_{2}}{B_{1}}-\frac{B_{1} c_{0}}{(1-\gamma)}+2 \mu c_{0} B_{1} \frac{(1-2 \gamma)}{(1-\gamma)^{2}}\right)\right]$,

$$
\begin{aligned}
\left|a_{1}-\mu a_{0}^{2}\right| \leq\left|-\frac{B_{1} c_{0}}{2(1-2 \gamma)}\right| & \\
& \cdot\left[\left|k_{1} \frac{c_{1}}{c_{0}}\right|+\left|k_{2}+k_{1}^{2}\left(\frac{B_{2}}{B_{1}}-\frac{B_{1} c_{0}}{(1-\gamma)}+2 \mu c_{0} B_{1} \frac{(1-2 \gamma)}{(1-\gamma)^{2}}\right)\right|\right] .
\end{aligned}
$$

Since $\varphi(\omega)$ is analytic and bounded in $U^{*}$ (see [25]), so we have

$$
\left|c_{n}\right| \leq 1-\left|c_{0}\right|^{2} \leq 1,(n>0) .
$$

By using this fact and the well-known inequality $\left|k_{1}\right| \leq 1$, we get

$$
\begin{aligned}
\left|a_{1}-\mu a_{0}^{2}\right| \leq & \frac{B_{1}}{2(1-2 \gamma)} \\
& \cdot\left[1+\left|k_{2}+k_{1}^{2}\left(\frac{B_{2}}{B_{1}}-\frac{B_{1} c_{0}}{(1-\gamma)}+2 \mu c_{0} B_{1} \frac{(1-2 \gamma)}{(1-\gamma)^{2}}\right)\right|\right] .
\end{aligned}
$$

Corollary 5. For $\varphi(\omega)=1$ and $\gamma=0$ in Theorem 4, we obtain the result by Silverman et al. [3] (see Theorem 4).

For $k(\omega)=\omega$ and repeating steps of Theorem 4 , we obtain the following corollary.

Corollary 6. Let $\lambda(\omega) \in \sum_{1}$ satisfies

$$
-\left[(1-\gamma)\left(\frac{\omega \lambda^{\prime}(\omega)}{\lambda(\omega)}\right)+\gamma\left(1+\frac{\omega \lambda^{\prime \prime}(\omega)}{\lambda^{\prime}(\omega)}\right)\right]-1<<\phi(\omega)-1, \quad\left(\omega \in U^{*}\right)
$$

then for any complex number $\mu$,

$$
\left|a_{1}-\mu a_{0}^{2}\right| \leq \frac{B_{1}}{2(1-2 \gamma)}\left[1+\left|\frac{B_{2}}{B_{1}}\right|+B_{1}\left|\frac{1}{1-\gamma}-2 \mu \frac{(1-2 \gamma)}{(1-\gamma)^{2}}\right|\right] .
$$

Theorem 7. Let $(\omega)=1+B_{1} \omega+B_{2} \omega^{2}+\cdots, B_{1}>0$, and $\varphi(\omega)$ $=c_{0}+c_{1} \omega+c_{2} \omega^{2}+\cdots$, if $\lambda(\omega), \delta(\omega)$, given by (1) and (5) be in the class $\sum_{q}^{M K}(\phi, \eta)$, and $\mu$ is a complex number, then

$$
\begin{aligned}
& \left|\frac{b_{1}+a_{1}}{2}-a_{1} \eta-\mu b_{0}^{2}\right| \\
& \cdot \leq \frac{B_{1}}{2}\left[1+\max \left\{1,\left|\frac{B_{2}}{B_{1}}\right|+B_{1}\left|\frac{1}{(-1-\eta)^{2}}-\frac{2 \mu}{(1-\eta)^{2}(-1-4 \eta)^{2}}\right|\right\}\right] .
\end{aligned}
$$

The inequalities is sharp for $k(\omega)=\omega$ or $k(\omega)=\omega^{2}$.

Proof. Let $\lambda(\omega), \delta(\omega) \in \sum_{q}^{M K}(\phi, \eta)$, then there exist analytic functions $\varphi(\omega)$ and $k(\omega)$, with $|\varphi(\omega)|<1, k(0)=0$, and $k(\omega)<1$ such that

$$
\frac{-\omega \lambda^{\prime}(\omega)}{(1-\eta) \delta(\omega)+\eta \omega \delta^{\prime}(\omega)}-1=\varphi(\omega)[\phi(k(\omega))-1], \quad\left(\omega \in U^{*}\right) .
$$

Taking first derivative of (1) and (5), and use in the left hand side of above equation, we obtain 


$$
\begin{aligned}
\frac{-\omega \lambda^{\prime}(\omega)}{(1-\eta) \delta(\omega)+\eta \omega \delta^{\prime}(\omega)} & =1+2 \eta(1+2 \eta)+b_{0}(1-\eta)(-1-4 \eta) \omega \\
& +\left[b_{0}^{2}(1-\eta)^{2}-b_{1}+2 a_{1} \eta-a_{1}\right] \omega^{2}+\cdots,
\end{aligned}
$$

then implies

$$
\begin{aligned}
\frac{-\omega \lambda^{\prime}(\omega)}{(1-\eta) \delta(\omega)+\eta \omega \delta^{\prime}(\omega)}-1= & 2 \eta(1+2 \eta)+b_{0}(1-\eta)(-1-4 \eta) \omega \\
& +\left[b_{0}^{2}(1-\eta)^{2}-b_{1}+2 a_{1} \eta-a_{1}\right] \omega^{2}+\cdots
\end{aligned}
$$

$$
\begin{aligned}
{[\phi(k(\omega))] } & =1+k_{1} B_{1} \omega+\left(k_{1}^{2} B_{2}+k_{2} B_{1}\right) \omega^{2} \\
& +\left(k_{3} B_{1}+2 k_{1} k_{2} B_{2}+k_{1}^{3} B_{3}\right) \omega^{3}+\cdots,
\end{aligned}
$$

which implies

$\varphi(\omega)[\phi(k(\omega))-1]=c_{0} k_{1} B_{1} \omega+\left(c_{0} k_{1}^{2} B_{2}+c_{0} k_{2} B_{1}+c_{1} k_{1} B_{1}\right) \omega^{2}+\cdots$

Comparing (26) and (28), we get

$$
\begin{gathered}
b_{0}=\frac{c_{0} k_{1} B_{1}}{(1-\eta)(-1-4 \eta)}, \\
\frac{b_{1}+a_{1}}{2}-a_{1} \eta=-\frac{B_{1} c_{0}}{2}\left[k_{2}+k_{1} \frac{c_{1}}{c_{0}}+k_{1}^{2}\left(\frac{B_{2}}{B_{1}}-\frac{B_{1} c_{0}}{(-1-4 \eta)^{2}}\right)\right] .
\end{gathered}
$$

Thus,

$$
\begin{aligned}
& \frac{b_{1}+a_{1}}{2}-a_{1} \eta-\mu b_{0}^{2}=-\frac{B_{1} c_{0}}{2} \\
& \cdot\left[k_{2}+k_{1} \frac{c_{1}}{c_{0}}+k_{1}^{2}\left(\frac{B_{2}}{B_{1}}-\frac{B_{1} c_{0}}{(-1-4 \eta)^{2}}+2 \mu \frac{c_{0} B_{1}}{(1-\eta)^{2}(-1-4 \eta)^{2}}\right)\right] .
\end{aligned}
$$
have

Since $\varphi(\omega)$ is analytic and bounded in $U^{*}$ (see [25]), we

$$
\left|c_{n}\right| \leq 1-\left|c_{0}\right|^{2} \leq 1, \quad(n>0) .
$$

By using this fact and the well-known inequality $\left|k_{1}\right| \leq 1$, we get

$$
\begin{aligned}
& \left|\frac{b_{1}+a_{1}}{2}-a_{1} \eta-\mu b_{0}^{2}\right| \\
& \quad \leq \frac{B_{1}}{2}\left[1+\left|k_{2}+k_{1}^{2}\left(\frac{B_{2}}{B_{1}}-\frac{B_{1} c_{0}}{(-1-4 \eta)^{2}}+2 \mu \frac{c_{0} B_{1}}{(1-\eta)^{2}(-1-4 \eta)^{2}}\right)\right|\right] .
\end{aligned}
$$

Corollary 8. For $\varphi(\omega)=1, \delta(\omega)=\lambda(\omega)$, and $\eta=0$ in Theorem 7, we obtain the result by Silverman et al. [3] (see Theorem 7). For $k(\omega)=\omega$ and repeating steps of Theorem 7 , we obtain the following corollary.
Corollary 9. Let $\lambda(\omega)$ and $\delta(\omega) \in \sum_{1}$ satisfy

$$
\frac{-\omega \lambda^{\prime}(\omega)}{(1-\eta) \delta(\omega)+\eta \omega \delta^{\prime}(\omega)}-1<<\phi(\omega)-1, \quad\left(\omega \in U^{*}\right),
$$

then for any complex number $\mu$,

$$
\begin{aligned}
& \left|\frac{b_{1}+a_{1}}{2}-a_{1} \eta-\mu b_{0}^{2}\right| \\
& \quad \leq \frac{B_{1}}{2}\left[1+\left|\frac{B_{2}}{B_{1}}\right|+B_{1}\left|\frac{1}{(-1-4 \eta)^{2}}-\frac{2 \mu}{(1-\eta)^{2}(-1-4 \eta)^{2}}\right|\right] .
\end{aligned}
$$

\section{Meromorphic Functions Related with the Bessel Function}

Let us consider the second order linear homogenous differential equation (see, Baricz [26])

$$
\omega^{2} k^{\prime \prime}(\omega)+\alpha \omega k^{\prime}(\omega)+\left[\beta \omega^{2}-\nu^{2}+(1-\alpha)\right] k(\omega)=0
$$

The function

$$
k_{v, \alpha, \beta}(\omega)=\sum_{t=0}^{\infty} \frac{(-\beta)^{t}}{\Gamma(t+1) \Gamma(t+v+1+(\alpha+1 / 2))}\left(\frac{\omega}{2}\right)^{2 t+v}
$$

is known as generalized Bessel's function of first kind and is the solution of differential equation given in (35). If we denote

$$
\begin{aligned}
\zeta_{v, \alpha, \beta} \lambda(\omega) & =\frac{2^{v} \Gamma(v+(\alpha+1 / 2))}{\omega^{(v / 2)+1}} k_{v, \alpha, \beta}\left(\omega^{1 / 2}\right), \\
& =\frac{1}{\omega}+\sum_{t=0}^{\infty} \frac{(-\beta)^{t+1} \Gamma(\nu+(\alpha+1 / 2))}{4^{t+1} \Gamma(t+2) \Gamma(t+v+1+(\alpha+1 / 2))}\left(\omega^{t}\right),
\end{aligned}
$$

where $\nu, \alpha$, and $\beta$ are positive real numbers. The operator $\zeta_{v, \alpha, \beta}$ is a meromorphic analogue introduced by Deniz [27] (see also Baricz et al. [28]) for analytic functions. In terms of convolution, $\zeta_{v, \alpha, \beta}$ is given by

$$
\begin{aligned}
\left(\zeta_{v, \alpha, \beta} \lambda\right)(\omega) & =k_{v, \alpha, \beta}(\omega) * \lambda(\omega) \\
& =\frac{1}{\omega}+\sum_{t=0}^{\infty} \frac{(-\beta)^{t+1} \Gamma(v+(\alpha+1 / 2))}{4^{t+1} \Gamma(t+2) \Gamma(t+v+1+(\alpha+1 / 2))} a_{t}(\omega)^{t} .
\end{aligned}
$$

The operator $\zeta_{v, \alpha, \beta}$ was introduced and studied by Mostafa et al. [29]. For more details, see [30, 31] and references cited therein. Motivated from the above cited work, we introduce the following classes of meromorphic functions.

Definition 10. A function $\lambda(\omega) \in \sum_{1}$ given by (1) is said to belong to the class $\sum_{\nu, \alpha, \beta}^{M C(q)}(\phi, \gamma)$ if

$$
-\left[(1-\gamma)\left(\frac{\omega\left(\zeta_{v, \alpha, \beta} \lambda\right)^{\prime}(\omega)}{\left(\zeta_{v, \alpha, \beta} \lambda\right)(\omega)}\right)+\gamma\left(1+\frac{\omega\left(\zeta_{v, \alpha, \beta} \lambda\right)^{\prime \prime}(\omega)}{\left(\zeta_{v, \alpha, \beta} \lambda\right)^{\prime}(\omega)}\right)\right]-1 \prec_{q} \phi(\omega)-1 .
$$


For $\gamma=0$, the class $\sum_{\nu, \alpha, \beta}^{M S(q)}(\phi)$ was studied by Zayed et al. [20].

Definition 11. Let $\sum_{v, \alpha, \beta}^{M K(q)}(\phi, \eta)$ be the subclasses of $\sum_{1}$ consisting of all functions $\lambda(\omega) \in \sum_{1}$ for which there exist $\delta(\omega)$ $\in \sum^{M S}(\alpha)$, and

$\frac{-\omega\left(\zeta_{v, \alpha, \beta} \lambda\right)^{\prime}(\omega)}{(1-\eta)\left(\zeta_{v, \alpha, \beta} \delta\right)(\omega)+\eta \omega\left(\zeta_{v, \alpha, \beta} \delta\right)^{\prime}(\omega)}-1 \prec_{q} \phi(\omega)-1 \quad\left(\omega \in U^{*}\right)$.

For $\eta=0$ and $\delta(\omega)=\lambda(\omega)$, the class $\sum_{v, \alpha, \beta}^{M S(q)}(\phi)$ was studied by Zayed et al. [20].

Theorem 12. Let $(\omega)=1+B_{1} \omega+B_{2} \omega^{2}+\cdots, B_{1}>0$, and $\varphi(\omega)=c_{0}+c_{1} \omega+c_{2} \omega^{2}+\cdots$, if $\lambda(\omega)$ given by (1) be in the class $\sum_{\nu, \alpha, \beta}^{M C(q)}(\phi, \gamma)$, and $\mu$ is a complex number, then

$$
\begin{aligned}
\left|a_{1}-\mu a_{0}^{2}\right| \leq & \frac{4^{2}(v+(\alpha+1 / 2))(v+1+(\alpha+1 / 2)) B_{1}}{\beta^{2}(1-2 \gamma)} \\
& \times\left[1+\max \left\{1,\left|\frac{B_{2}}{B_{1}}\right|+B_{1}\left|1-\mu\left(\frac{(v+(\alpha+1 / 2))(1-2 \gamma)}{(v+1+(\alpha+1 / 2))(1-\gamma)}\right)\right|\right\}\right] .
\end{aligned}
$$

Proof. Let $\lambda(\omega) \in \sum_{v, \alpha, \beta}^{M C(q)}(\phi, \gamma)$, then there exist analytic functions $\varphi(\omega)$ and $k(\omega)$, with $|\varphi(\omega)|<1, k(0)=0$, and $k(\omega)<1$ such that

$$
\begin{aligned}
& -\left[(1-\gamma)\left(\frac{\omega\left(\zeta_{v, \alpha, \beta} \lambda\right)^{\prime}(\omega)}{\left(\zeta_{v, \alpha, \beta} \lambda\right)(\omega)}\right)+\gamma\left(1+\frac{\omega\left(\zeta_{v, \alpha, \beta} \lambda\right)^{\prime \prime}(\omega)}{\left(\zeta_{v, \alpha, \beta} \lambda\right)^{\prime}(\omega)}\right)\right]-1 \\
& =\varphi(\omega)[\phi(k(\omega))-1] .
\end{aligned}
$$

Taking first and second derivative of (38), in use of the left side of the above equation, we obtain

$$
\begin{aligned}
& -\left[(1-\gamma)\left(\frac{\omega\left(\zeta_{v, \alpha, \beta} \lambda\right)^{\prime}(\omega)}{\left(\zeta_{v, \alpha, \beta} \lambda\right)(\omega)}\right)+\gamma\left(1+\frac{\omega\left(\zeta_{v, \alpha, \beta} \lambda\right)^{\prime \prime}(\omega)}{\left(\zeta_{v, \alpha, \beta} \lambda\right)^{\prime}(\omega)}\right)\right] \\
& =(1-\gamma)\left[1+\frac{\beta a_{0} \omega}{4(\nu+(\alpha+1 / 2))}\right. \\
& \quad-\frac{2 \beta^{2} a_{1} \omega^{2}}{4^{2} \times 2(v+1+(\alpha+1 / 2))(v+(\alpha+1 / 2))} \\
& \left.+\frac{\beta^{2} a_{0}^{2} \omega^{2}}{4^{2}(v+(\alpha+1 / 2))^{2}}+\cdots\right] \\
& +\gamma\left[1+\frac{2 \beta^{2} a_{1} \omega^{2}}{4^{2} \times 2(v+1+(\alpha+1 / 2))(v+(\alpha+1 / 2))}+\cdots\right],
\end{aligned}
$$

$$
\begin{aligned}
& -\left[(1-\gamma)\left(\frac{\omega\left(\zeta_{v, \alpha, \beta} \lambda\right)^{\prime}(\omega)}{\left(\zeta_{v, \alpha, \beta} \lambda\right)(\omega)}\right)+\gamma\left(1+\frac{\omega\left(\zeta_{v, \alpha \beta} \lambda\right)^{\prime \prime}(\omega)}{\left(\zeta_{\nu, \alpha, \beta} \lambda\right)^{\prime}(\omega)}\right)\right]-1 \\
& =\frac{\beta a_{0}}{4(v+(\alpha+1 / 2))}(1-\gamma) \omega \\
& \quad+\left[\frac{\beta^{2} a_{0}^{2}}{4^{2}(v+(\alpha+1 / 2))^{2}}(1-\gamma)\right. \\
& \left.\quad-\frac{2 \beta^{2} a_{1}}{4^{2} \times 2(v+1+(\alpha+1 / 2))(v+(\alpha+1 / 2))}(1-2 \gamma)\right] \omega^{2}+\cdots, \\
& {[\phi(k(\omega))]=1+k_{1} B_{1} \omega+\left(k_{1}^{2} B_{2}+k_{2} B_{1}\right) \omega^{2}} \\
& \quad+\left(k_{3} B_{1}+2 k_{1} k_{2} B_{2}+k_{1}^{3} B_{3}\right) \omega^{3}+\cdots,
\end{aligned}
$$

which implies

$$
\varphi(\omega)[\phi(k(\omega))-1]=c_{0} k_{1} B_{1} \omega+\left(c_{0} k_{1}^{2} B_{2}+c_{0} k_{2} B_{1}+c_{1} k_{1} B_{1}\right) \omega^{2}+\cdots .
$$

Comparing (44) and (46), we get

$$
\begin{aligned}
a_{0}= & \frac{4(\nu+(\alpha+1 / 2)) c_{0} k_{1} B_{1}}{\beta(1-\gamma)}, \\
a_{1}= & -\frac{4^{2}(\nu+1+(\alpha+1 / 2))(\nu+(\alpha+1 / 2)) B_{1}}{\beta^{2}(1-2 \gamma)} \\
& \cdot\left[c_{0} k_{2}+c_{1} k_{1}+k_{1}^{2}\left(\frac{c_{0} B_{2}}{B_{1}}-\frac{c_{0} B_{1}}{(1-\gamma)}\right)\right] .
\end{aligned}
$$

Thus,

$$
\begin{gathered}
a_{1}-\mu a_{0}^{2}=-\frac{4^{2}(\nu+1+(\alpha+1 / 2))(\nu+(\alpha+1 / 2)) B_{1}}{\beta^{2}(1-2 \gamma)} \\
\times\left[c_{0} k_{2}+k_{1}^{2}\left\{\frac{c_{1}}{k_{1}}+\frac{c_{0} B_{2}}{B_{1}}-B_{1}\left(\frac{c_{0}}{(1-\gamma)}-\frac{(\nu+(\alpha+1 / 2))(1-2 \gamma) c_{0}}{(v+1+(\alpha+1 / 2))(1-\gamma)}\right)\right\}\right], \\
\left|a_{1-} \mu a_{0}^{2}\right| \leq\left|-\frac{4^{2}(\nu+1+(\alpha+1 / 2))(v+(\alpha+1 / 2)) B_{1}}{\beta^{2}(1-2 \gamma)}\right| \\
\times\left[\left|c_{0} k_{2}\right|+\left|k_{1}^{2}\left\{\frac{c_{1}}{k_{1}}+\frac{c_{0} B_{2}}{B_{1}}-B_{1}\left(\frac{c_{0}}{(1-\gamma)}-\frac{(v+(\alpha+1 / 2))(1-2 \gamma) c_{0}}{(v+1+(\alpha+1 / 2))(1-\gamma)}\right)\right\}\right|\right]
\end{gathered}
$$

Since $\varphi(\omega)$ is analytic and bounded in $U^{*}$ (see [25]), we have $\left|c_{n}\right| \leq 1-\left|c_{0}\right|^{2} \leq 1,(n>0)$.

By using this fact and the well-known inequality $\left|k_{1}\right| \leq 1$, we get

$$
\begin{gathered}
\left|a_{1}-\mu a_{0}^{2}\right| \leq \frac{4^{2}(v+(\alpha+1 / 2))(v+1+(\alpha+1 / 2)) B_{1}}{\beta^{2}(1-2 \gamma)} \\
\times\left[1+\max \left\{1,\left|\frac{B_{2}}{B_{1}}\right|+B_{1}\left|1-\mu\left(\frac{(v+(\alpha+1 / 2))(1-2 \gamma)}{(v+1+(\alpha+1 / 2))(1-\gamma)}\right)\right|\right\}\right] .
\end{gathered}
$$

thus implies
We have thus completed the proof of Theorem 12 . 
For $k(\omega)=\omega$ and repeating steps of Theorem 12, we obtain the following corollary.

Corollary 13. Let $\lambda(\omega) \in \sum_{1}$ satisfies

$$
-\left[(1-\gamma)\left(\frac{\omega\left(\zeta_{v, \alpha, \beta} \lambda\right)^{\prime}(\omega)}{\left(\zeta_{v, \alpha, \beta} \lambda\right)(\omega)}\right)+\gamma\left(1+\frac{\omega\left(\zeta_{v, \alpha, \beta} \lambda\right)^{\prime \prime}(\omega)}{\left(\zeta_{v, \alpha, \beta} \lambda\right)^{\prime}(\omega)}\right)\right]-1 \ll \phi
$$$$
\cdot(\omega)-1\left(\omega \in U^{*}\right) \text {. }
$$

Then, for any complex number $\mu$,

$$
\begin{aligned}
\left|a_{1}-\mu a_{0}^{2}\right| \leq & \frac{4^{2}(\nu+(\alpha+1 / 2))(\nu+1+(\alpha+1 / 2)) B_{1}}{\beta^{2}(1-2 \gamma)} \\
& \cdot\left[1+\left|\frac{B_{2}}{B_{1}}\right|+B_{1}\left|\frac{1}{(1-\gamma)}-\mu\left(\frac{(\nu+(\alpha+1 / 2))(1-2 \gamma)}{(\nu+1+(\alpha+1 / 2))}\right)\right|\right] .
\end{aligned}
$$

Theorem 14. Let $\phi(\omega)=1+B_{1} \omega+B_{2} \omega^{2}+\cdots, B_{1}>0$, and $\varphi$ $(\omega)=c_{0}+c_{1} \omega+c_{2} \omega^{2}+\cdots$, if $\lambda(\omega)$ and $\delta(\omega)$ given by (1) and (5) be in the class $\sum_{v, \alpha, \beta}^{M K(q)}(\phi, \eta)$ and $\mu$ is a complex number, then

$$
\begin{gathered}
\left|\frac{b_{1}+a_{1}}{2}-b_{1} \eta-\mu b_{0}^{2}\right| \leq \frac{4^{2}(\nu+(\alpha+1 / 2))(\nu+1+(\alpha+1 / 2)) B_{1}}{\beta^{2}} \\
\times\left[1+\max \left\{1,\left|\frac{B_{2}}{B_{1}}\right|+B_{1}\left|1-\mu\left(\frac{(\nu+(\alpha+1 / 2))}{(\nu+1+(\alpha+1 / 2))(1-\eta)^{2}}\right)\right|\right\}\right] .
\end{gathered}
$$

Proof. Let $\lambda(\omega)$ and $\delta(\omega) \in \sum_{v, \alpha, \beta}^{M K(q)}(\phi, \eta)$ then there exist analytic functions $\varphi(\omega)$ and $k(\omega)$, with $|\varphi(\omega)|<1, k(0)=0$, and $k(\omega)<1$ such that

$$
\frac{-\omega\left(\zeta_{v, \alpha, \beta} \lambda\right)^{\prime}(\omega)}{(1-\eta)\left(\zeta_{v, \alpha, \beta} \delta\right)(\omega)+\eta \omega\left(\zeta_{v, \alpha, \beta} \delta\right)^{\prime}(\omega)}-1=\varphi(\omega)[\phi(k(\omega))-1]
$$

Taking first derivative of (5) and (38) in use of the left side of above equation, we have

$$
\begin{aligned}
& \frac{-\omega\left(\zeta_{v, \alpha, \beta} \lambda\right)^{\prime}(\omega)}{(1-\eta)\left(\zeta_{v, \alpha, \beta} \delta\right)(\omega)+\eta \omega\left(\zeta_{v, \alpha, \beta} \delta\right)^{\prime}(\omega)} \\
& =1+\frac{\beta b_{0}(1-\eta)}{4(v+(\alpha+1 / 2))} \omega \\
& \quad+\left(\frac{\beta^{2} b_{0}^{2}(1-\eta)^{2}}{4^{2}(v+(\alpha+1 / 2))^{2}}-\frac{\beta^{2} b_{1}(1-2 \eta)}{4^{2} \times 2(v+1+(\alpha+1 / 2))(v+(\alpha+1 / 2))}\right. \\
& \left.\quad-\frac{\beta^{2} a_{1}}{4^{2} \times 2(v+1+(\alpha+1 / 2))(v+(\alpha+1 / 2))}\right) \omega^{2}+\cdots,
\end{aligned}
$$

and this implies

$$
\begin{aligned}
& \frac{-\omega\left(\zeta_{v, \alpha, \beta} \lambda\right)^{\prime}(\omega)}{(1-\eta)\left(\zeta_{v, \alpha, \beta} \delta\right)(\omega)+\eta \omega\left(\zeta_{v, \alpha, \beta} \delta\right)^{\prime}(\omega)}-1 \\
& =\frac{\beta b_{0}(1-\eta)}{4(v+(\alpha+1 / 2))} \omega \\
& \quad+\left(\frac{\beta^{2} b_{0}^{2}(1-\eta)^{2}}{4^{2}(v+(\alpha+1 / 2))^{2}}-\frac{\beta^{2} b_{1}(1-2 \eta)}{4^{2} \times 2(v+1+(\alpha+1 / 2))(v+(\alpha+1 / 2))}\right. \\
& \left.\quad-\frac{\beta^{2} a_{1}}{4^{2} \times 2(v+1+(\alpha+1 / 2))(v+(\alpha+1 / 2))}\right) \omega^{2}+\cdots,
\end{aligned}
$$

$$
\begin{aligned}
{[\phi(k(\omega))] } & =1+k_{1} B_{1} \omega+\left(k_{1}^{2} B_{2}+k_{2} B_{1}\right) \omega^{2} \\
& +\left(k_{3} B_{1}+2 k_{1} k_{2} B_{2}+k_{1}^{3} B_{3}\right) \omega^{3}+\cdots
\end{aligned}
$$

which implies

$\varphi(\omega)[\phi(k(\omega))-1]=c_{0} k_{1} B_{1} \omega+\left(c_{0} k_{1}^{2} B_{2}+c_{0} k_{2} B_{1}+c_{1} k_{1} B_{1}\right) \omega^{2}+\cdots$

Comparing (55) and (57), we get

$$
\begin{gathered}
b_{0}=\frac{4(\nu+(\alpha+1 / 2)) c_{0} k_{1} B_{1}}{\beta(1-\eta)} \omega, \\
\frac{b_{1}+a_{1}}{2}-b_{1} \eta=-\frac{4^{2}(\nu+(\alpha+1 / 2))(\nu+1+(\alpha+1 / 2)) B_{1}}{\beta^{2}} \\
\cdot\left[c_{0} k_{2}+c_{1} k_{1}+k_{1}^{2}\left(\frac{c_{0} B_{2}}{B_{1}}-c_{0}^{2} B_{1}\right)\right] .
\end{gathered}
$$

Thus,

$\frac{b_{1}+a_{1}}{2}-b_{1} \eta-\mu b_{0}^{2}=-\frac{4^{2}(\nu+(\alpha+1 / 2))(\nu+1+(\alpha+1 / 2)) B_{1}}{\beta^{2}}$

$\times\left[c_{0} k_{2}+c_{1} k_{1}+k_{1}^{2}\left(\frac{c_{0} B_{2}}{B_{1}}-c_{0}^{2} B_{1}+\mu \frac{(\nu+(\alpha+1 / 2)) c_{0}^{2} B_{1}}{(\nu+1+(\alpha+1 / 2))(1-\eta)^{2}}\right)\right]$,

$\left|\frac{b_{1}+a_{1}}{2}-b_{1} \eta-\mu b_{0}^{2}\right| \leq\left|-\frac{4^{2}(\nu+(\alpha+1 / 2))(\nu+1+(\alpha+1 / 2)) B_{1}}{\beta^{2}}\right|$

$\times\left[\left|c_{0} k_{2}\right|+\left|k_{1}^{2}\left\{c_{0} k_{2}+\frac{c_{0} B_{2}}{B_{1}}-B_{1}\left(c_{0}^{2}-\mu \frac{(v+(\alpha+1 / 2)) c_{0}^{2}}{(v+1+(\alpha+1 / 2))(1-\eta)^{2}}\right)\right\}\right|\right]$. have

Since $\varphi(\omega)$ is analytic and bounded in $U^{*}$ (see [25]), we

$$
\left|c_{n}\right| \leq 1-\left|c_{0}\right|^{2} \leq 1, \quad(n>0) .
$$

By using this fact and the well-known inequality, $\left|k_{1}\right| \leq 1$, we get 


$$
\begin{aligned}
& \left|\frac{b_{1}+a_{1}}{2}-b_{1} \eta-\mu b_{0}^{2}\right| \leq \frac{4^{2}(v+(\alpha+1 / 2))(v+1+(\alpha+1 / 2)) B_{1}}{\beta^{2}} \\
\times & {\left[1+\max \left\{1,\left|\frac{B_{2}}{B_{1}}\right|+B_{1}\left|1-\mu\left(\frac{(v+(\alpha+1 / 2))}{(v+1+(\alpha+1 / 2))(1-\eta)^{2}}\right)\right|\right\}\right] . }
\end{aligned}
$$

We have thus completed the proof of Theorem 14 .

For $k(\omega)=\omega$ and repeating steps of Theorem 14, we obtain the following corollary.

Corollary 15. Let $\lambda(\omega)$ and $\delta(\omega)$ satisfy

$\frac{-\omega\left(\zeta_{v, \alpha, \beta} \lambda\right)^{\prime}(\omega)}{(1-\eta)\left(\zeta_{\nu, \alpha, \beta} \delta\right)(\omega)+\eta \omega\left(\zeta_{\nu, \alpha, \beta} \delta\right)^{\prime \prime}(\omega)}-1<<\phi(\omega)-1, \quad\left(\omega \in U^{*}\right)$

then for any complex number $\mu$,

$$
\begin{gathered}
\left|\frac{b_{1}+a_{1}}{2}-b_{1} \eta-\mu b_{0}^{2}\right| \leq \frac{4^{2}(\nu+(\alpha+1 / 2))(v+1+(\alpha+1 / 2)) B_{1}}{\beta^{2}} \\
\times\left[1+\left|\frac{B_{2}}{B_{1}}\right|+B_{1}\left|1-\mu\left(\frac{(\nu+(\alpha+1 / 2))}{(\nu+1+(\alpha+1 / 2))(1-\eta)^{2}}\right)\right|\right] .
\end{gathered}
$$

\section{Conclusion}

In our present investigation, we have defined and systematically studied the famous Fekete-szego inequality for some subclass of meromorphic functions by using quasisubordination. It is important to mention that certain results in the literature, for example $[3,19,20]$, are special cases of the results obtained by us.

\section{Data Availability}

No data is used.

\section{Conflicts of Interest}

The authors declare that they have no conflict of interest.

\section{Authors' Contributions}

SH came with the main thoughts and helped to draft the manuscript. SGAS and AR proved the main theorems. ZS and $\mathrm{MD}$ revised the paper. All authors read and approved the final manuscript.

\section{Funding}

The fifth author is supported by GUP-2019-032.

\section{References}

[1] M. I. S. Robertson, "On the theory of univalent functions," Annals of Mathematics, vol. 37, no. 2, p. 374, 1936.
[2] W. Kaplan, "Close-to-convex schlicht functions," Michigan Mathematical Journal, vol. 1, no. 2, pp. 169-185, 1952.

[3] H. Silverman, K. Suchithra, B. A. Stephen, and A. Gangadharan, "Coefficient bounds for certain classes of meromorphic Functions," Journal of Inequalities and Applications, vol. 2008, no. 1, Article ID 931981, 2008.

[4] M. K. Aouf, A. O. Mostafa, and H. M. Zayed, "Convolution properties for some subclasses of meromorphic functions of complex order," Abstract and Applied Analysis, vol. 2015, Article ID 973613, 6 pages, 2015.

[5] M. S. Robertson, "Quasi-subordination and coefficient conjectures," Bulletin of the American Mathematical Society, vol. 76, no. 1, pp. 1-10, 1970.

[6] S. P. Goyal and P. Goswami, "Majorization for certain classes of meromorphic functions defined by integral operator," Annales UMCS, Mathematica, vol. 66, no. 2, pp. 57-62, 2012.

[7] N. E. Cho and S. Owa, "Sufficient conditions for meromorphic starlikeness and close-to-convexity of order $\alpha$," International Journal of Mathematics and Mathematical Sciences, vol. 26, Article ID 630710, 3 pages, 2001.

[8] J. Clunie, "On meromorphic schlicht functions," Journal of the London Mathematical Society, vol. s1-34, no. 2, pp. 215-216, 1959.

[9] M. Darus, S. Hussain, M. Raza, and J. Sokol, "On a subclass of starlike functions," Results in Mathematics, vol. 73, no. 1, p. 22, 2018.

[10] S. Hussain, A. Rasheed, M. A. Zaighum, and M. Darus, "A subclass of analytic functions related tok-uniformly convex and starlike functions," Journal of Function Spaces, vol. 2017, Article ID 9010964, 7 pages, 2017.

[11] J. L. Liu, H. M. Srivastava, and Y. Yuan, "A family of meromorphically multivalent functions which are starlike with respect to k-symmetric points," Journal of Mathematical Inequalities, vol. 11, no. 3, pp. 781-798, 2007.

[12] J. Miller, "Convex meromorphic mappings and related functions," Proceedings of the American Mathematical Society, vol. 25, no. 2, pp. 220-228, 1970.

[13] S. Mahmood, M. Jabeen, S. N. Malik, H. M. Srivastava, R. Manzoor, and S. M. J. Riaz, "Some coefficient inequalities of q-starlike functions associated with conic domain defined by q-derivative," Journal of Function Spaces, vol. 2018, Article ID 8492072, 13 pages, 2018.

[14] C. Pommerenke, "On meromorphic starlike functions," Pacific Journal of Mathematics, vol. 13, no. 1, pp. 221-235, 1963.

[15] J. Sokol and M. Nunokawa, "On some class of convex functions," Comptes Rendus Mathematique, vol. 353, no. 5, pp. 427-431, 2015.

[16] H. M. Srivastava, S. Altinkaya, and S. Yalcin, "Certain subclasses of bi-univalent functions associated with the Horadam polynomials," Iranian Journal of Science and Technology, Transactions A: Science, vol. 43, no. 4, pp. 1873-1879, 2019.

[17] B. A. Uralegaddi and A. R. Desai, "Integrals of meromorphic starlike functions with positive and fixed second coefficients," Indian Academy of Mathematics, vol. 24, no. 1, pp. 27-36, 2002.

[18] A. Rasheed, S. Hussain, S. Ghoos Ali Shah, M. Darus, and S. Lodhi, "Majorization problem for two subclasses of meromorphic functions associated with a convolution operator," AIMS Mathematics, vol. 5, no. 5, pp. 5157-5170, 2020. 
[19] M. H. Mohd and M. Darus, "Fekete-Szegö problems for quasisubordination classes," Abstract and Applied Analysis, vol. 2012, Article ID 192956, 14 pages, 2012.

[20] H. M. Zayed, S. Bulut, and A. O. Mostafa, "Quasi-subordination and coefficient bounds for certain classes of meromorphic functions of complex order," Communications Faculty Of Science University of Ankara Series A1Mathematics and Statistics, vol. 68, no. 1, pp. 1197-1205, 2018.

[21] F. R. Keogh and E. P. Merkes, "A coefficient inequality for certain classes of analytic functions," Proceedings of the American Mathematical Society, vol. 20, no. 1, pp. 8-12, 1969.

[22] M. K. Aouf, R. M. El-Ashwah, and H. M. Zayed, "Fekete-Szegö inequalities for certain class of meromorphic functions," Journal of the Egyptian Mathematical Society, vol. 21, no. 3, pp. 197-200, 2013.

[23] S. Hussain, H. M. Srivastava, A. Raziq, and M. Raza, "The Fekete-Szego functional for subclass of analytic functions associated with quasi-subordination," Carpathian Journal of Mathematics, vol. 34, pp. 103-113, 2018.

[24] A. O. Mostafa, H. M. Srivastava, M. K. Aouf, and H. M. Zayed, "Basic and fractional $q$-calculus and associated Fekete-Szego problem for $p$-valently $q$-starlike functions and $p$-valently qconvex functions of complex order," Miskolc Mathematical Notes, vol. 20, no. 1, pp. 489-509, 2019.

[25] Z. Nehari, Conformal Mapping, McGraw-Hill, New York, 1952.

[26] A. Baricz, "Generalized Bessel functions of the first kind," in Lecture Notes in Math, vol. 1994, Springer-Verlag, Berlin, 2010.

[27] E. Deniz, "Differential subordination and super ordination results for an operator associated with the generalized Bessel function," Preprint.

[28] Á. Baricz, E. Deniz, M. Çağlar, and H. Orhan, "Differential subordinations involving generalized Bessel functions," Bulletin of the Malaysian Mathematical Sciences Society, vol. 38, no. 3, pp. 1255-1280, 2015.

[29] A. O. Mostafa, M. K. Aouf, and H. M. Zayed, "Convolution properties for some subclasses of meromorphic bounded functions of complex order," International Journal of Open Problems in Complex Analysis, vol. 8, no. 2, pp. 12-19, 2016.

[30] H. M. Srivastava, S. Abbas, S. Tyagi, and D. Lassoued, "Global exponential stability of fractional-order impulsive neural network with time-varying and distributed delay," Mathematical Methods in the Applied Sciences, vol. 41, no. 5, pp. 20952104, 2018.

[31] B. S. Alkahtani, S. Mustafa, and T. Bulboacă, "Subclasses of analytic functions defined by generalized hypergeometric functions," Journal of Function Spaces, vol. 2016, Article ID 7390410, 6 pages, 2016. 